\title{
INTERVENÇÕES PREVENTIVAS DE ENFERMAGEM EM CASOS DE CÂNCER PÓS- TRANSPLANTE RENAL: UMA REVISÃO BIBLIOGRÁFICA
}

\author{
Preventive intervention of nursing in cancer cases after kidney transplantation: \\ a literature review
}

Livia Nunes Rodrigues Leme'; Silvana Soares dos Santos ${ }^{2}$

\section{RESUMO}

Introdução: Devido ao aumento da sobrevida do paciente transplantado renal e maior exposição aos agentes imunossupressores, as neoplasias tornaram-se uma das três principais causas de morte após o transplante na última década, sendo, portanto, cada vez mais importante, em todos os programas de transplante renal, a prevenção e cuidado com as mesmas. Objetivos: realizar um levantamento bibliográfico sobre o desenvolvimento de neoplasia em pacientes transplantados renais e identificar as intervenções preventivas de enfermagem em relação ao desenvolvimento de neoplasias em transplantados renais. Métodos: Pesquisa do tipo bibliográfica, com coleta dos dados de publicações impressas e fontes eletrônicas, nos bancos de dados BIREME e PubMed. A pesquisa inicial resultou em 2724 resumos, onde, após verificação dos critérios de inclusão e exclusão propostos, foram selecionados 79 artigos para leitura completa. A amostra final contou com 40 artigos e seis fontes impressas, que foram avaliados e analisados. Conclusão: Através deste estudo puderam ser observadas questões importantes relacionadas ao desenvolvimento, em pacientes transplantados renais, de diversos tipos de neoplasias, principalmente neoplasias incomuns na população geral, como tumores cutâneos do tipo epidermoide, Sarcoma de Kaposi, desordens linfoproliferativas, carcinoma cervical e colorretal, e neoplasias mais comuns, como mama e próstata. Diante disso, conclui-se que a monitorização desses pacientes quanto a manifestações precoces de acometimento de neoplasias deve fazer parte da rotina da equipe de enfermagem e multiprofissional. O enfermeiro deve priorizar ações que visem a prevenção, promoção da saúde e detecção precoce de complicações em receptores de transplante renal, proporcionando oportunidades para o ensino e aprendizagem durante o acompanhamento pré e pós-transplante, o que pode contribuir para diminuição do risco de desenvolvimento de neoplasia nessa clientela.

Descritores: Transplante Renal; Neoplasia; Cuidados de Enfermagem.

Instituição:

${ }^{1}$ Internal Medicine Postgraduate Program, Medical School Federal University of Minas Gerais, Belo Horizonte, MG, Brazil.

${ }^{2}$ Medical School - Federal University of Minas Gerais, Belo

Horizonte, MG, Brazil

\section{Correspondência:}

Wanessa Trindade Clemente

Av.Alfredo Balena s $/ \mathrm{n}^{\circ}, 4^{\circ}$ and, sala 403, CEP 30130-100, Faculdade de Medicina - Depto.Propedêutica Complementar, Belo Horizonte/MG Phone: +55 (31) 3409-9774 Fax: +55 (31) 3409-9774

E-mail: wanclemente@yahoo.com.br

\section{INTRODUÇÃO}

Segundo a Associação Brasileira de Transplantes de Órgãos, o Brasil possui um dos maiores programas públicos de transplante de órgãos do mundo, sendo o segundo país com maior número de transplantes, atrás apenas dos Estados Unidos. ${ }^{1}$

No caso dos transplantes renais, estes constituem o tratamento mais adequado para a doença renal crônica, devido à melhora da sobrevida do paciente, de sua qualidade de vida e ainda por reduzir os custos do tratamento, quando comparado com a diálise. ${ }^{2}$ Entretanto, é de fundamental importância a realização do acompanhamento ambulatorial pela equipe multiprofissional, a fim de prevenir e tratar precocemente qualquer complicação. 
Em relação a essas complicações, as neoplasias tornaram-se uma das três principais causas de morte após o transplante na última década e é, portanto, cada vez mais importante a prevenção e o cuidado com as mesmas, em todos os programas de transplante renal. ${ }^{3}$

Calcula-se que pacientes transplantados possuam risco de acometimento neoplásico até cem vezes maior em relação à população geral, e tais tumores tendem a apresentar comportamento mais agressivo, com maior potencial de progressão, invasão, recorrência local e metastatização. ${ }^{4}$

Devido a toda complexidade do transplante renal, essa modalidade terapêutica exige que a equipe multiprofissional, inclusive a equipe de enfermagem, preste assistência específica, com qualidade e domínio técnico-científico, para embasar a sua atuação. ${ }^{5}$ No processo de transplante renal, a Enfermagem desempenha papel fundamental, pois participa de forma atuante, desde a captação do órgão até o acompanhamento pós-cirúrgico ambulatorial. A assistência ao paciente transplantado renal é altamente especializada, requerendo a atuação do enfermeiro nas diversas fases do processo e exigindo pessoal com capacitação específica.

Mediante o exposto, esta pesquisa apresenta como objetivos: Realizar um levantamento bibliográfico sobre o desenvolvimento de neoplasias em pacientes transplantados renais; Identificar as intervenções preventivas de enfermagem em relação ao desenvolvimento de neoplasias em transplantados renais.

\section{MÉTODOS}

O presente estudo tem abordagem qualitativa e utilizou a pesquisa do tipo bibliográfica para alcançar os objetivos propostos. A coleta dos dados deu-se através de levantamento bibliográfico, publicações impressas e fontes eletrônicas, nos bancos de dados BIREME e PubMed.

Para a busca eletrônica, os descritores utilizados foram: transplante renal, neoplasia e cuidados de enfermagem, em diferentes combinações. Os critérios de inclusão da pesquisa foram: estudos sobre diferentes tipos de neoplasia pós-transplante de órgãos e renal, estudos que relacionavam os cuidados de enfermagem ao paciente transplantado renal e estudos que abordavam ações preventivas de enfermagem relacionadas a neoplasia. Foram considerados artigos publicados nos últimos cinco anos.

Foram considerados critérios de exclusão textos em idiomas diversos do português, inglês ou espanhol; textos que não estavam disponíveis na íntegra gratuitamente nas bases de dados; textos que abordavam exclusivamente relatos de caso.

A busca inicial resultou em 2724 resumos e, aplicados os critérios de inclusão e exclusão efetuou-se a leitura completa de 79 artigos, dos quais 39 foram excluídos por não atenderem aos objetivos da pesquisa. A amostra final contou, portanto, com 40 artigos, que foram avaliados e analisados.

Além de fontes eletrônicas foram utilizadas ainda seis fontes impressas, a saber: duas dissertações; um livro; uma publicação estatística e dois protocolos de ações.

Figura 1- Desenho do estudo

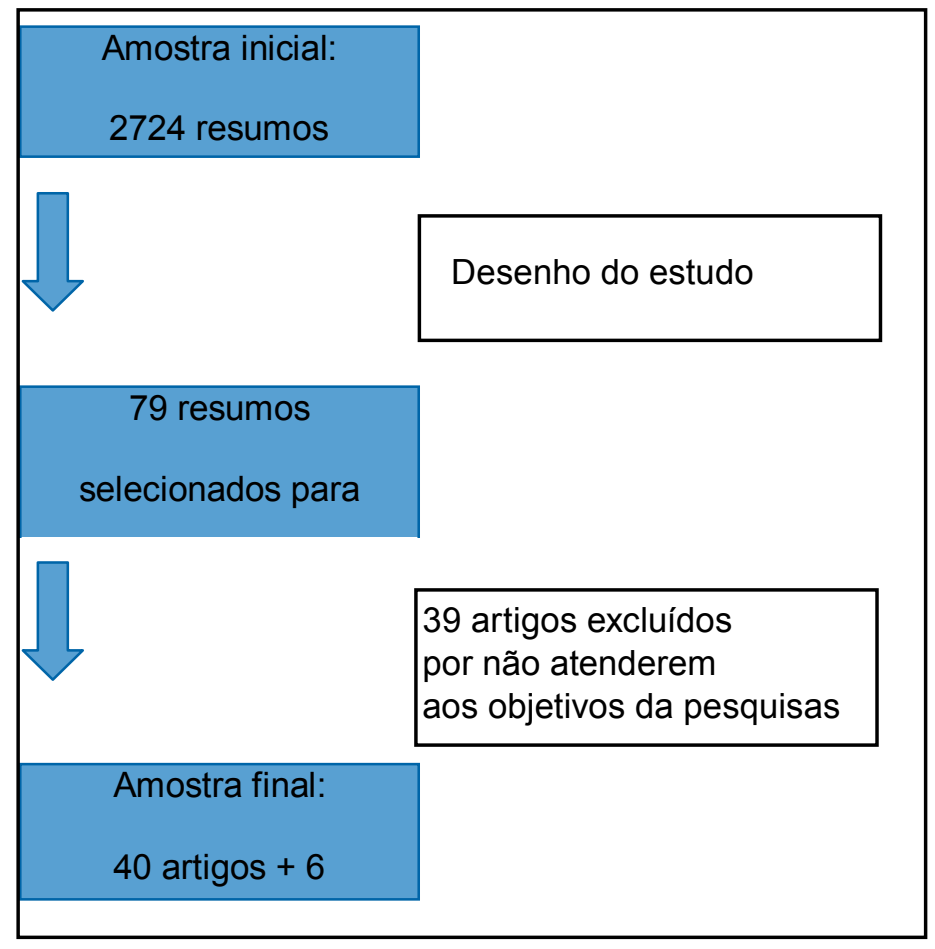

\section{REVISÃO DA LITERATURA}

\section{Incidência de neoplasias pós-transplante renal}

A melhora dos regimes imunossupressores, o melhor controle das complicações infecciosas e o melhor conhecimento dos sistemas de histocompatibilidade, além do aperfeiçoamento na captação, distribuição de órgãos e nas técnicas de transplante renal têm aumentado a sobrevida dos pacientes transplantados, praticamente dobrando sua expectativa de vida. ${ }^{6}$ Entretanto, essa modalidade terapêutica possui riscos inerentes, entre eles, maior risco para neoplasia após o transplante. ${ }^{7}$

As neoplasias malignas respondem pela terceira maior causa de mortalidade entre pacientes transplantados 
renais, perdendo somente para as infecções e as enfermidades cardiovasculares. ${ }^{4} \mathrm{~A}$ incidência de mortes por doenças cardiovasculares e infecções em países desenvolvidos está reduzindo, como resposta a uma melhor triagem, diagnóstico, prevenção e abordagens de intervenção. Em contraste, a detecção precoce, prevenção e tratamento da neoplasia pós-transplante renal ainda não são bem compreendidos, com evidências escassas para apoiar a triagem de rotina, a gestão de fatores de risco e as terapias de intervenção. ${ }^{8}$ Pesquisas afirmam que, com a terapia imunossupressora com duração superior a 10 anos, a incidência de tumor maligno poderá atingir o índice de $20 \%$. As neoplasias poderão então substituir as doenças cardiovasculares e tornar-se a maior ameaça em longo prazo (> 10 anos) na sobrevida de pacientes transplantados renais. ${ }^{9} \mathrm{~A}$ mortalidade relacionada a neoplasias poderá se tornar a primeira causa de morte dentro das próximas duas décadas. ${ }^{10}$

A incidência de neoplasia pós-transplante renal é certamente maior se comparada à população geral, devido ao tratamento imunossupressor, onde se verifica o aumento substancial de tumores raros na população geral. Os tipos mais comuns são a neoplasia de pele, desordens linfoproliferativas e sarcoma de Kaposi, considerados os mais frequentes. Há também maior incidência de neoplasia renal, vulvovaginal e de colo uterino, bexiga, faringe, laringe, cavidade oral, olho, hepatocarcinoma e outros sarcomas. , $9,11,12^{2}$

Os riscos são especialmente altos para neoplasias provocadas por infecções virais, incluindo o linfoma não-Hodgkin e linfoma de Hodgkin (devido ao vírus de Epstein-Barr [EBV]), o sarcoma de Kaposi (vírus do herpes humano 8), neoplasias anogenitais e cervical (papilomavírus humano - HPV) e neoplasia de fígado (hepatite $\mathrm{C}$ e vírus $\mathrm{B}) .^{13}$

Em contrapartida, as taxas de incidência de neoplasia da mama e da próstata na população transplantada são similares às observadas na população em geral ou mesmo reduzidas. ${ }^{12}$ Pacientes com risco para neoplasia tornam-se inelegíveis para o transplante renal e o rastreio pré-transplante pode ser a causa da diminuição da incidência dessas neoplasias em transplantados renais, apesar dessa conclusão não estar ainda muita clara. $^{7}$

As taxas de sobrevida de pacientes transplantados renais também são diferenciadas. Dados do Israel Penn Internacional Transplant Tumour Registry (IPITTR), afirmam que a sobrevivência para certos tipos de neoplasia, como de cólon, pulmão, mama, próstata e bexiga, foi significativamente menor em pacientes transplantados, em comparação aos da população geral, independentemente do estágio histológico no momento do diagnóstico. As taxas de sobrevida dos transplantados em estágio avançado de neoplasia colorretal, próstata e pulmão foram $10 \%$, 40\%, e $20 \%$, respectivamente, em comparação com 40\%, 80\%, 30\% na população em geral. Mesmo para os pacientes com doença em estágio inicial, os prognósticos globais são piores do que para pacientes da população geral. ${ }^{8}$

As razões para esse aumento na incidência de neoplasia em pacientes transplantados renais são multifatoriais, incluindo exposições ambientais, dietas, ocupações, comportamentos de saúde, genética e nível socioeconômico; a interação de fatores relacionados ao transplante, tais como os tipos, duração e carga de imunossupressão pré e pós-transplante, a exposição a infecções virais específicas, tempo em diálise antes do transplante, e fatores de risco tradicionais, como a exposição ao sol, tabagismo e idade.,

A rapidez com que algumas neoplasias se desenvolvem após o transplante renal sugere que a oncogênese viral possa estar relacionada ao início da imunossupressão, que pode promover replicação viral latente. Pacientes com falha de transplante renal e consequente redução ou cessação da imunossupressão têm o risco de desenvolvimento de neoplasia relacionado a vírus diminuído e retornando aos níveis observados no pré-transplante. ${ }^{3} \mathrm{O}$ padrão de ocorrência de algumas neoplasias durante os períodos de função do enxerto renal e de diálise, após falha do transplante, difere e sugere que o efeito da imunossupressão no risco de neoplasia seria completamente reversível. A incidência de sarcoma de Kaposi e linfoma não Hodgkin, neoplasia de lábio e melanoma diminui acentuadamente quando ocorre a reinstituição da diálise. No entanto, existem poucos dados sobre o risco da neoplasia ser ou não reduzido quando a imunossupressão cessa. ${ }^{14}$

O risco geral de neoplasia em pacientes transplantados renais é moderado pela duração e intensidade da imunossupressão e não por componentes individuais do regime de drogas. Várias pesquisas não conseguiram demostrar de forma consistente a associação do aumento do risco de neoplasia em pacientes transplantados renais com determinada droga. É possível que existam pequenas diferenças de efeito entre as drogas, mas essas diferenças são compensadas pelos efeitos maiores de outros fatores de risco conhecidos para a neoplasia. ${ }^{2,3}$

Por outro lado, algumas drogas imunossupressoras, como o sirolimos, por exemplo, têm efeito imunossupressor e antitumor. Diversos estudos têm demostrado menor incidência de malignidades em pacientes transplantados renais cuja imunossupressão é realizada com inibidores de mTOR, que com inibidores de calcineurina. Uma 
importante vantagem do uso de inibidores de mTOR pode ser a proteção rejeição imunológica do aloenxerto, enquanto simultaneamente impede desenvolvimento dae neoplasia em grupos de alto risco..$^{10}$

Independente da ação individual ou não de determinadas drogas, o consenso é que a toxicidade dos imunossupressores, de forma geral, e sua relação com o desenvolvimento de neoplasia pós-transplante renal tornou-se um problema e sua monitorização terapêutica passou a ocupar lugar imprescindível no bom prognóstico dos procedimentos pós-enxerto.

\section{PRINCIPAIS NEOPLASIAS EM TRANSPLANTADOS RENAIS E SUAS CAUSAS}

\section{Tumores cutâneos}

A neoplasia da pele é comumente dividida em não melanoma (carcinoma basocelular - CBC - e carcinoma epidermoide ou espinocelular - CEC) e melanoma. $O$ não melanoma é o mais incidente no país e apresenta altos índices de cura com baixa taxa de mortalidade. Já o melanoma, responsável por aproximadamente $5 \%$ dos casos de neoplasia da pele, apresenta alta letalidade, principalmente pela sua alta capacidade de desenvolvimento de metástases. ${ }^{15}$

O período de latência médio entre o transplante renal e o aparecimento da primeira lesão neoplásica de pele é muito variável na literatura. ${ }^{16} \mathrm{O}$ não melanoma é a neoplasia mais comum que ocorre após o transplante, com um período de latência de 5-8 anos. Cerca de metade dos pacientes transplantados renais com uma neoplasia de pele não melanoma primário desenvolve uma segunda neoplasia do mesmo tipo. ${ }^{12}$

A incidência de CEC é cerca de 40 a 250 vezes maior, quando comparada com a população em geral, podendo acometer cerca de 34 a $48 \%$ de indivíduos no período de 15 a 20 anos após o transplante. ${ }^{6}$ O CBC mostrase mais frequente que o CEC nos primeiros anos póstransplante renal e, com o decorrer dos anos, essa relação inverte-se nesse grupo de pacientes. ${ }^{6,17}$

Os fatores de risco para o desenvolvimento de neoplasia de pele são semelhantes entre pacientes transplantados renais e a população em geral, como exposição aos raios ultravioletas, pessoas com pele e olhos claros, história de exposição e queimaduras graves de pele, características genéticas individuais, raça, idade, o uso de diferentes regimes imunossupressores $e$ as infecções virais, como as infecções por HPV que, em interação com os raios ultravioletas, citocinas e proteínas da célula hospedeira, contribui para a oncogênese..$^{16,18}$
O processo de desenvolvimento de neoplasia em pacientes transplantados renais pode ser acelerado por dois mecanismos distintos dos imunossupressores: ação cancerígena direta desses agentes e vigilância imunológica e erradicação de lesões pré-cancerosas prejudicadas devido à imunossupressão permanente. ${ }^{19}$

Foram encontradas evidências de que o tratamento com Azatioprina (Aza) está fortemente associado ao risco de desenvolvimento de CEC pós-transplante e que esse risco era proporcional ao aumento acumulado da dose, isto é, o risco era maior com a duração mais longa do tratamento. Em contraste, a Ciclosporina (CsA) não foi associada ao aumento do risco de CEC pós-transplante. Entretanto, podem haver diferenças importantes nesse risco específico conferido pelas drogas devido a interações de diversos outros fatores que contribuem para o desenvolvimento de neoplasias. ${ }^{20}$

Para pacientes transplantados renais com neoplasia de pele é aconselhável a suspensão da azatioprina, considerada como o principal agente envolvido na gênese desses tumores, mantendo-se esquema duplo com ciclosporina ou tacrolimo e prednisona ou com associação de sirolimo ou everolimo. ${ }^{11}$

Além do risco aumentado de neoplasias de pele em pacientes transplantados renais, esses tumores, nesse grupo de pacientes, costumam ser mais agressivos, com idade mais precoce ao diagnóstico, doença com maior potencial de invasão, aumento do risco de recorrência local, altas taxas de múltiplas lesões primárias, aumento da tendência de metástases regionais e à distância e maior taxa de mortalidade quando comparados com às neoplasias de pele em pacientes da população geral. ${ }^{6,18}$

\section{Sarcoma de Kaposi}

Trata-se de um tumor de pele de origem multicêntrica, caracterizado histologicamente por espaços vasculares revestidos por endotélio e células fusiformes. Pode apresentar-se com lesões únicas ou múltiplas em superfícies mucosas, incluindo a pele, pulmões, trato gastrointestinal e tecido linfoide. ${ }^{21}$

O desenvolvimento de SK está associado à presença do vírus HHV-8 latente em receptores de transplante renal, no entanto, a presença do vírus não confirma o desenvolvimento da doença, pois existe a necessidade de reativação do $\mathrm{HHV}-8$, que pode ser desencadeada pela resposta imunológica comprometida. ${ }^{22}$

A incidência de SK em pacientes transplantados renais varia de acordo com a localização geográfica, sendo mais frequente no Oriente Médio e na Arábia Saudita (5\%), em comparação aos EUA e à Austrália 
$(0,25$ a $0,5 \%) \cdot{ }^{23}$ A prevalência do SK em receptores de transplante de órgãos varia entre 0,5 e $5 \%$, mas sobe para $23 \%$ em doentes que eram positivos para HHV-8 antes do transplante. ${ }^{22}$

A incidência de SK (fortemente relacionado com a imunossupressão, tanto em receptores de transplante como em pessoas infectadas com HIV) diminuiu acentuadamente na reinstituição da diálise, em caso de falha do enxerto. ${ }^{14}$

O SK não foi significativamente relacionado à utilização de imunossupressores específicos, e há ainda algumas dúvidas sobre o papel da intensidade da imunossupressão no seu desenvolvimento. Entretanto, alguns estudos mostram que a incidência do SK foi enormemente aumentada, após a introdução de ciclosporina. A regressão das lesões mucocutâneas pode ser obtida através da redução ou mesmo a retirada imunossupressão e há alto risco de recorrência após o retransplante em pacientes que tiveram remissão clínica do SK com redução / retirada de imunossupressão em um transplante anterior. ${ }^{21,22}$

\section{Desordens linfoproliferativas pós-transplante (DLPT)}

As desordens linfoproliferativas são caracterizadas por proliferação anormal do tecido linfoide que ocorre em transplantados de órgãos sólidos ou de células hematopoiéticas, devido à imunossupressão. Estão entre as complicações malignas mais graves e potencialmente fatais do transplante renal. Essas malignidades são predominantemente associadas às infecções pelo vírus de Epstein-Barr, em $80 \%$ a $90 \%$ dos casos. Aproximadamente $90 \%$ da população adulta têm evidências sorológicas de exposição prévia a EBV e o vírus permanece no indivíduo infectado de forma latente, podendo ser reativado devido à imunossupressão. Entretanto, casos de DLPT não associados ao vírus EBV também têm sido descritos e podem ter sua incidência aumentada. ${ }^{24}$

Alguns estudos demonstram que, no transplante renal, o risco para DLPT chegou a ser cerca de 40 vezes maior do que na população em geral. Uma vez que essa complicação se desenvolve, ela é associada a uma taxa significativamente elevada de morbidade e mortalidade, o que mostra que a morte ocorre em mais de $50 \%$ dos pacientes. ${ }^{25}$

O risco está também relacionado ao tipo de órgão transplantado. Estudos mostram o transplante de intestino como o de mais elevada incidência (19\% - 30\%) contra $1 \%-3 \%$ do transplante renal, o que confirma essa diferenciação de incidência da DLPT quanto aos tipos de órgão transplantados, mostrando que ela é elevada em transplantados renais quando comparada à população em geral, porém menor quando comparada aos transplantes de outros órgãos. Apesar da aparente baixa incidência, o número absoluto de casos de DLPT em transplantados renais é bastante elevado, devido ao número relativamente alto de transplantes renais e da longa sobrevida desses pacientes.24 A incidência da DLPT eleva-se imediatamente após o transplante, diminuindo posteriormente, em seguida, e subindo novamente após 4-5 anos de transplante. ${ }^{26}$

Em relação ao tratamento, nem sempre a DLPT localizada requer terapia sistêmica, pois a cirurgia ou radioterapia pode ser usada nesses pacientes, com lesão cutânea ou uma única lesão gastrintestinal. Além disso, as terapias locais usadas em conjunto com a redução da imunossupressão têm melhores resultados, diminuindo a mortalidade. A redução da imunossupressão em pacientes transplantados renais a doses que permitam a restauração da resposta imune contra o linfoma sem causar rejeição de enxerto, é bem tolerada. ${ }^{24}$

\section{Neoplasia Cervical}

O transplante renal aumenta a incidência de neoplasia intraepitelial cervical de 14 a 16 vezes e a de neoplasia cervical invasivo de 3,0 a 8,6 vezes. A imunossupressão parece não causar a progressão do carcinoma em si, mas está associada ao início dos estágios de displasia, devido a defeitos nos mecanismos responsáveis pela eliminação do HPV. O maior risco de neoplasia cervical em pacientes transplantadas renais refere-se à replicação desse vírus oncogênico. ${ }^{27}$

O HPV é conhecido como a principal causa de carcinoma cervical e de neoplasia intra-epitelial cervical (NIC). Mais de 100 tipos de HPV são conhecidos e, entre esses, 15 são classificados como tipos de HPV de alto risco. ${ }^{28}$ Receptoras de transplante renal diagnosticadas com infecção por HPV de alto risco requerem acompanhamento atento, pois uma simples inflamação genital pode ser responsável pela persistência da infecção por HPV, facilitando, assim, a progressão de lesões pré-malignas da cérvix. ${ }^{29}$

As vacinas contra o HPV têm-se mostrado eficazes na prevenção de neoplasia do colo do útero na população geral, porém foram encontradas respostas abaixo do ideal quanto à eficácia da vacina em receptores de transplante. Esses fatores devem ser considerados na tomada de decisão sobre fornecer ou não a vacina para receptores de transplantes. Muitos centros transplantadores podem escolher prescrever a vacinação para pessoas de qualquer idade, candidatas a transplante, ou para os receptores pós-transplante. No entanto, a imunogenicidade dessa vacina no póstransplante de órgãos ainda não está esclarecida. ${ }^{30}$ 
Uma análise dos fatores clínicos relacionados ao carcinoma cervical que se desenvolve após o transplante renal e uma análise do prognóstico desse carcinoma são necessárias, uma vez que existem muitas questões sobre o tema a ser esclarecidas e poucos estudos referentes à temática. ${ }^{28}$

É importante citar ainda que, além do HPV, outros fatores de risco estão relacionados à neoplasia cervical, como início precoce das atividades sexuais, multiplicidade de parceiros, parceiro sexual masculino com múltiplas parceiras, tabagismo e outras infecções genitais de repetição, não somente as viróticas. ${ }^{31}$

\section{Neoplasia Renal}

Estudos sugerem aumento da incidência de neoplasia renal em transplantados renais aumentada de 16 a 24 vezes quando comparado à população geral. ${ }^{32} \mathrm{Em}$ outro estudo, a neoplasia renal foi o tipo de neoplasia mais frequente, com incidência de $6,8 \%$ de todos os tumores. ${ }^{33}$

A incidência dessas neoplasias varia de $1 \%$ a $5 \%$ e esses tumores podem surgir devido à transmissão pelo doador, condições referentes ao receptor após o transplante, ou por falha no enxerto. A neoplasia pode ocorrer tanto no rim nativo quanto no transplantado. No Cincinnati Transplant Tumor Registry descobriram que as neoplasias renais representaram $4,6 \%$ de todas as neoplasias que ocorrem em receptores de transplantes. Entre esses tipos de neoplasia, apenas $10 \%$ ocorreram no enxerto renal. ${ }^{34}$

O risco elevado de neoplasia renal entre os receptores de rim é bem descrito. Alguns casos iniciais surgem como um resultado da transformação maligna de cistos que se desenvolvem em rins em fase terminal antes do transplante. ${ }^{13} \mathrm{O}$ tempo em diálise, a presença de doença renal cística adquirida e a doença renal subjacente são fatores de risco para o desenvolvimento da neoplasia, tanto no rim nativo quanto no transplantado. ${ }^{32,33}$

O tempo entre a data do transplante e o desenvolvimento do carcinoma renal em rins transplantados é extremamente variável, entre nove e 228 meses. Um curto período de tempo, entre a transplantação e o diagnóstico enxerto de tumor no enxerto do rim, pode sugerir que o mesmo foi transmitido a partir do doador. ${ }^{34}$ É possível que efeitos nefrotóxicos ou diretamente cancerígenos de alguns imunossupressores possam contribuir para neoplasias que surgem no rim transplantado. Entretanto, estudos demostraram maior ausência de risco de neoplasia de rim em pessoas infectadas pelo HIV, o que argumenta contra um papel importante da condição de imunossuprimido, colocando apenas os efeitos nefrotóxicos e cancerígenos dos imunossupressores em questão. ${ }^{13}$
Sobre o tratamento, existem alguns estudos que citam a cirurgia conservadora (nefrectomia parcial do enxerto) como um procedimento seguro e eficaz, obtendo a preservação da função renal e controle da neoplasia em curto prazo. No entanto, mesmo sendo viável a cirurgia conservadora, ela é tecnicamente mais difícil, devendo sempre considerar que o que se quer é preservar a função renal do enxerto. ${ }^{32}$ Estudos apontam que, após a nefrectomia parcial ou tratamento minimamente invasivo, o tratamento imunossupressor foi mantido em $83 \%$ dos pacientes, não sendo necessárias modificações da terapia imunossupressora. ${ }^{34}$

\section{Neoplasia Colorretal}

Alguns estudos apontam que em transplantados renais o risco para neoplasia colorretal é semelhante à população geral ou ligeiramente aumentado. ${ }^{7}$ Em contraste, outros autores citam que o risco de desenvolvimento de neoplasia colorretal é elevado entre os receptores de transplante renal e está associado com a duração da imunossupressão, independentemente da idade. ${ }^{35}$ Muitos transplantados que desenvolvem neoplasia colorretal são mais jovens no momento do diagnóstico e têm piores prognósticos quando comparados com a população em geral. ${ }^{36}$

Atualmente, há evidências consistentes que mostram maior risco de incidência de neoplasia colorretal em transplantados renais, de pelo menos duas a três vezes. Para os homens transplantados renais com neoplasia colorretal, a sobrevida em cinco anos é de $27 \%$, enquanto que na população geral, com a neoplasia mas sem transplantes, é de $75 \%{ }^{3,8} \mathrm{O}$ intervalo médio de tempo entre o transplante renal e o desenvolvimento de neoplasia colorretal é de mais desuperior a 10 anos após o transplante. ${ }^{37}$

O EBV é considerado fator causal de algumas malignidades gastrointestinais, especialmente neoplasia gástrica, e tem-se observado que a patogênese de lesões colorretais pode envolver fatores virológicos. $O$ Citomegalovírus (CMV) também pode estar envolvido na carcinogênese e progressão do carcinoma colorretal. ${ }^{35}$ Além desses fatores, importantes em transplantados renais, podemos citar outros fatores de risco, como idade (prevalente em pessoas acima de 50 anos), presença de pólipos, história de neoplasia anterior, história familiar, história de enterocolite ulcerativa e constipação intestinal crônica, tabagismo e sedentarismo. ${ }^{31}$

\section{Neoplasia de Mama}

Apesar de ser tão comum na população geral, a incidência de neoplasia de mama em transplantados renais é baixa, indicando que o risco aumenta muito 
modestamente após o transplante. ${ }^{38}$ Apesar de, aparentemente, a imunossupressão não aumentar a incidência de neoplasia de mama em transplantados renais, pode aumentar sua agressividade, quando ela ocorre. ${ }^{39}$

Dados do Registro de Diálise e Transplante da Austrália e Nova Zelândia, afirmam que, apesar da menor incidência, as mulheres transplantadas com neoplasia de mama têm mortalidade $40 \%$ maior em comparação com as mulheres com neoplasia de mama na população geral. ${ }^{8}$ Transplantados renais que desenvolvem neoplasia de mama são muitas vezes mais jovens no momento do diagnóstico e têm pior prognóstico do que a população geral. Entretanto, quando o diagnóstico precoce é feito, o prognóstico do paciente é excelente, se o tratamento for administrado durante as fases iniciais da doença. ${ }^{39}$

O rastreio da neoplasia de mama deve ser verificado caso a caso. A American Society of Transplantation (AST) e a European Best Practice Guidelines (EBPG), por exemplo, recomendam o rastreamento da neoplasia de mama em todos as transplantadas entre 50 e 69 anos de idade e, para as mulheres transplantadas entre $40 \mathrm{e}$ 49 anos de idade, pode ser anual ou bienal.

Contudo, mulheres transplantadas submetidas à triagem por mamografia devem estar cientes do potencial para resultado falso positivo, que chega a $35 \%$, particularmente em mulheres que desenvolveram múltiplos adenomas mamários benignos pelo uso da ciclosporina em longo prazo. Resultados falso positivos levarão a procedimentos diagnósticos desnecessários e exaustivos, como a biópsia com agulha fina, por exemplo. ${ }^{3,39}$

O tratamento da neoplasia de mama na população transplantada é um pouco complicado por alguns fatores, como a determinação da dose correta do imunossupressor, sobrevida do enxerto e doses adjuvante de quimioterapia. A imunossupressão é uma área preocupante, porque há poucos dados disponíveis para orientar as decisões e, quando a cirurgia é necessária, o acesso à diálise deve ser considerado. ${ }^{40}$

A função do enxerto renal deve ser considerada antes da quimioterapia, pois alguns agentes quimioterápicos podem afetar função renal. O Paclitaxel pode ser opção para os doentes com função do enxerto prejudicada. ${ }^{39}$

\section{A atuação da Enfermagem}

A contribuição do enfermeiro para o resultado favorável do transplante renal é inegável e a complexidade do cuidado tem se tornado cada vez maior. O enfermeiro que atua em transplantes presta cuidado especializado na proteção, promoção e reabilitação da saúde de candidatos, receptores e seus familiares. Tal cuidado inclui prevenção, detecção precoce, tratamento e reabilitação de pacientes com problemas de saúde relacionados às doenças prévias ao transplante ou comorbidades associadas ao tratamento pós-transplante. ${ }^{41}$

O principal papel do enfermeiro em uma unidade de transplante renal é promover maior adesão ao tratamento, com o seguimento das orientações educacionais, principalmente aquelas relacionadas à identificação precoce de complicações. ${ }^{42}$ Cabe ainda à enfermagem, proporcionar a educação em saúde ao paciente, cuidador e familiar ainda no pré-operatório, a fim de expor todas as variáveis relevantes do pré e pósoperatório. ${ }^{43}$

A sistematização da assistência de enfermagem para o paciente transplantado renal contribui para a organização do trabalho do enfermeiro, para a redução do risco de complicações e para a melhoria da qualidade de vida, pois fornece meios para propor intervenções e direcioná-las às necessidades do cliente. ${ }^{44}$

Durante a consulta de enfermagem, tanto ambulatorial quanto na internação hospitalar, o enfermeiro, entre outras medidas, deve executar ações de prevenção primária e secundária no controle da neoplasia no paciente transplantado renal. As ações de prevenção primária dizem respeito às orientações alimentares; a cessação do tabagismo; o papel das atividades físicas como fator protetor contra a neoplasia; a diminuição ou cessação da ingestão de álcool; a exposição solar e o papel das infecções no desenvolvimento de alguns tipos de neoplasia. Sobre as ações de prevenção secundária são descritos os meios de detecção precoce de neoplasias, como o rastreamento ou screening e o diagnóstico precoce. ${ }^{31}$

Portanto, os elementos-chave para a atuação dos enfermeiros no cuidado do paciente transplantado renal e na prevenção de neoplasias nessa clientela incluem: a educação de pacientes, familiares e cuidadores; a implementação de intervenções que mantenham ou melhorem a saúde fisiológica, psicológica e social, como o acompanhamento periódico, realização de exames pré e pós-transplante renal, triagem de pacientes com histórico de neoplasias, estabelecimento de um plano de cuidados individualizados; o uso de intervenções que facilitem e promovam mudanças de comportamento e adesão ao tratamento, como a abordagem dos meios de prevenção das neoplasias em geral; bem como, dar suporte aos pacientes e familiares no planejamento, implementação e avaliação do cuidado. ${ }^{41}$ 


\section{DISCUSSÃO}

Considerando os objetivos do estudo, foram abordados assuntos referentes ao desenvolvimento de neoplasias em pacientes transplantados renais, incluindo as neoplasias mais comuns e com incidência mais elevada que na população em geral e neoplasias com menor incidência na população de pacientes transplantados renais, mas também com importância clínica devido à alta taxa de mortalidade. Foram abordados ainda assuntos referentes às intervenções preventivas de enfermagem em relação ao desenvolvimento de neoplasias em transplantados renais. Algumas neoplasias de incidência elevada ou moderadamente elevada em pacientes transplantados renais, como anogenital, hepatocelular e lábio, por exemplo, não foram abordadas devido à falta da literatura consistente, mediante os critérios de inclusão e exclusão propostos.

Abordadas as questões sobre as neoplasias citadas na revisão de literatura, é importante falar ainda mais profundamente sobre as intervenções preventivas de enfermagem em relação ao desenvolvimento de neoplasias em transplantados renais.

O significado do transplante renal pode representar, na percepção do próprio paciente e sua família, melhorias na qualidade de vida, porém é de extrema importância destacar para os mesmos que há uma série de cuidados de saúde que necessitam ser gerenciados. ${ }^{45}$ O transplante renal pode ser entendido erroneamente por alguns pacientes, como uma cura definitiva de sua doença, levando-os a negligenciar comportamentos que são essenciais para o sucesso do procedimento. ${ }^{42}$ Assim, é preciso realizar o levantamento dos diagnósticos de enfermagem inerentes a cada paciente, bem como estabelecer um plano individual de cuidados, que buscará atender a todas as necessidades do paciente, cuidadores e familiares, visando a melhora da assistência prestada. ${ }^{43}$

A abordagem de doenças malignas pós-transplante renal deve começar com medidas de prevenção geral, pré e pós-transplante. Para reduzir a morbidade e mortalidade entre os pacientes transplantados renais, o diagnóstico precoce e tratamento de malignidades é extremamente importante. A prevenção das doenças malignas nessa clientela é ainda um desafio, que pode ser superado através da identificação precoce dos fatores de risco. ${ }^{25}$

No pré-transplante, por exemplo, deve-se fazer a triagem de pacientes com histórico de neoplasia. Existem recomendações atuais para adiar o transplante em caso de neoplasia prévia, pois existe a possibilidade de recorrência. As recomendações citadas variam entre os tipos e estágios da neoplasia e as características do paciente, mas, em média, são de dois a cinco anos para a maioria das neoplasias de órgãos sólidos. ${ }^{8}$ Além da verificação da ocorrência de neoplasia prévia, os futuros receptores de transplante renal, que estão infectados pelo $\mathrm{EBV}$, vírus das hepatites $\mathrm{B}$ e $\mathrm{C}, \mathrm{HPV}$ e outros vírus potencialmente oncogênicos, devem ser orientados pela equipe multiprofissional, incluindo a equipe de enfermagem, sobre a possibilidade de ocorrência de neoplasias pós-transplante. ${ }^{9}$

Em relação a alguma diferenciação de rastreio da neoplasia para os transplantados renais, não foram encontrados estudos consistentes que mostrem evidências dessa diferenciação, apesar dessa população ser considerada de risco para a ocorrência de diversos tipos de neoplasia. Apesar de o rastreio ser recomendado para todos os indivíduos com transplantes, essas recomendações são na sua maioria verificadas a partir da população em geral e não com um foco no transplantado renal. ${ }^{8}$

Considerando, portanto, essas recomendações e sabendo da relação entre imunossupressão, infecção viral e neoplasia, além dose fatores de risco conhecidos e outros ainda não esclarecidos relacionados à carcinogênese, é necessário um acompanhamento criterioso dos pacientes transplantados renais, através de exames periódicos, para detectar precocemente uma possível neoplasia. A equipe de enfermagem deve estar atenta e, em conjunto com a equipe multiprofissional, adotar medidas de prevenção, detecção precoce e tratamento para essas complicações, pois isso é essencial para inibir e/ou minimizar os danos causados por essas patologias.

$\mathrm{Na}$ sequência são descritas as questões preventivas específicas para as neoplasias citadas, que podem e devem ser abordadas pelos profissionais de enfermagem no cuidado aos pacientes transplantados.

Em relação à neoplasia de pele, a prevenção deve ser considerada uma das intervenções primordiais para esses pacientes, visto ser essa a mais comum na população transplantada renal, a fim de detectar precocemente lesões pré-malignas e prevenir o desenvolvimento da neoplasia. A vigilância e a educação para a prevenção são essenciais nesses pacientes, uma vez que, segundo pesquisas, há risco elevado de desenvolvimento de um segundo tumor cutâneo nos primeiros cinco anos após o diagnóstico do primeiro..$^{17}$ Os pacientes devem ser orientados quanto à diminuição de exposição solar e o uso de filtros solares. É preciso ainda orientá-los quanto à observação do surgimento de lesões suspeitas para avaliação e encaminhamento, caso necessário. O encaminhamento ao dermatologista é fundamental, quando se verifica 
o surgimento dessas lesões suspeitas. O tratamento precoce evitará complicações e sofrimento físico e psíquico do paciente. ${ }^{31}$

Nenhum estudo abordou alguma forma de prevenção ao sarcoma de Kaposi e apenas um estudo abordou a prevenção em relação às DLPTs.

Ainda existem controvérsias sobre o método de vigilância, pois ainda não há consenso sobre como determinar a positividade do EBV. Entretanto, embora possam ser menos confiáveis em pacientes em uso de medicações imunossupressoras, os testes sorológicos de antígeno da cápsula viral do EBV ou anticorpos contra antígenos nucleares são considerados mais específicos. Todos esses marcadores aumentam antes de um diagnóstico de DLPT, ocorrendo um pico em torno do tempo de diagnóstico. Sendo assim, sua monitorização pode favorecer o diagnóstico e tratamento precoces, diminuindo as altas taxas de mortalidade e melhorando a sobrevida do paciente, porém ainda há necessidade de estudos do tema. ${ }^{24}$

Quanto à prevenção da neoplasia cervical, a triagem precoce é útil e métodos de rastreio para a população geral têm sido amplamente aplicados em pacientes que receberam um transplante renal. No entanto, nenhum método de rastreio adequado tem sido estabelecido para esses pacientes, pois pouco se sabe sobre a taxa de progressão do carcinoma de colo do útero e o tempo de progressão em pacientes transplantados renais.

As sociedades americana e europeia de transplantes renais recomendam teste de Papanicolau e exame pélvico, conforme as recomendações brasileiras. ${ }^{28,31}$

Além disso, o desenvolvimento de vacinas contra o vírus HPV tem demonstrado que esse pode ser um instrumento poderoso na prevenção das neoplasias associadas a esses vírus, entretanto, as pesquisas mostraram uma resposta abaixo do ideal, portanto, estudos mais aprofundados sobre a eficácia dessas vacinas em pacientes transplantados renais devem ser realizados e sua utilização pré e pós-transplante deve ser discutida caso a caso. ${ }^{30}$

As atividades educativas para prevenção de neoplasia cervical devem ser voltadas à sensibilização de mulheres com vida sexual ativa, principalmente as que estão na faixa etária entre 25 e 60 anos, para a realização do exame Papanicolau. ${ }^{31}$

Sobre a neoplasia renal, apenas um estudo cita meios de prevenção, de acordo com as diretrizes da Associação Europeia de Urologia (EAU), onde os pacientes de transplante renal devem seguir controles periódicos de ultrassom em ambos os rins, tanto o nativo quanto $o$ transplantado. ${ }^{32}$
Quanto à neoplasia colorretal, as recomendações para seu rastreio estão longe de serem padronizadas. Um estudo cita recomendações variadas, como as da American Society of Transplantation (AST) com teste anual de sangue oculto nas fezes e sigmoidoscopia a cada cinco anos. Na Austrália, a triagem do sangue oculto é bienal, recomendada pela National Health and Medical Research Counci (NHMRC). Já na Europa, a sugestão da European Best Practice Guidelines for Renal Transplantation (EBPG) é o rastreio anual para todos os receptores de transplante usando o sangue oculto nas fezes e todos os testes positivos precisam ser seguido por colonoscopias diagnósticas. ${ }^{3}$

O paciente deve ser orientado na consulta de enfermagem quanto à identificação de fatores de risco, como alterações no hábito intestinal, presença de sangue nas fezes, diarreia ou constipação, fezes mais finas que o usual, fadiga e perda de peso. Devem ser observados ainda os exames de sangue que demonstrem anemia, sem sinais de sangramento aparente em indivíduos acima de 50 anos. As orientações também devem ser direcionadas a uma dieta rica em frutas e verduras e baixa ingestão de carnes vermelhas, o que pode ajudar a reduzir o risco para a neoplasia colorretal. Alguns estudos mostraram que a suplementação de ácido fólico e cálcio também ajuda a reduzir o risco. ${ }^{31}$

O diagnóstico precoce da neoplasia colorretal é extremamente importante, porém muitas vezes é dificultado pela ocorrência tardia desses sintomas, e pelo preconceito da população quanto aos métodos de diagnóstico, como o toque retal, a sigmoidoscopia e a colonoscopia, além do teste de sangue oculto nas fezes, entretanto, as práticas educativas relacionadas à prevenção devem ser sempre abordadas.

Quantoa neoplasia de mama, apesar daimunossupressão não aumentar sua incidência, pode ocorrer aumento de sua agressividade biológica e, portanto, a detecção precoce pode ser de extrema importância para o diagnóstico da doença numa fase inicial, para iniciar a terapia adequada, melhorando a sobrevida e qualidade de vida do paciente. ${ }^{39}$

A consulta de enfermagem, em relação a essa neoplasia, deve incluir: a identificação dos fatores de risco com base na anamnese, realização de exame clínico das mamas, orientação sobre observação de alterações nas mamas, exame mamográfico, autoexame das mamas realizado periodicamente pela própria mulher, encaminhamento adequado de casos classificados como de "risco alto" ou com anormalidades, nesse caso, independentemente do risco. ${ }^{31}$ Da mesma forma, a Association of Women's Health, Obstetric and Neonatal Nurses (AWHONN) acredita que é importante que enfermeiros e outros 
prestadores de cuidados de saúde realizem o rastreio da neoplasia de mama com um plano individualizado. Enfermeiros que trabalham com mulheres devem ser capazes de educá-las sobre a anatomia normal da mama, anormalidades, fatores de risco da neoplasia de mama, além dos benefícios, limitações e riscos das técnicas de rastreio. ${ }^{46}$

Independente das questões relacionadas ao rastreio das neoplasias colorretal e de mama, vistas anteriormente, no Brasil as recomendações quanto ao rastreio nos de pacientes transplantados renais permanecem as mesmas da população em geral. ${ }^{31}$

A consulta de enfermagem para os pacientes transplantados renais deve ainda abordar todos os meios de prevenção relacionados às neoplasias em geral, como a cessação do tabagismo, que é principal fator de risco evitável, não só da neoplasia, mas também de doenças cardiovasculares e respiratórias; a alimentação saudável, pobre em gorduras saturadas e rica em frutas, legumes e verduras; o controle do peso, já que o sobrepeso e a obesidade podem influenciar no desenvolvimento de neoplasias; a prática de atividade física, conforme orientação médica; a cessação do consumo de álcool, que também é fator de risco para diversas neoplasias. ${ }^{31}$

Diante de todas essas questões, é extremamente importante que os enfermeiros envolvidos no processo de transplante renal examinem continuamente sua prática profissional e busquem meios de melhorar a assistência de enfermagem prestada a essa clientela, através da educação continuada.

O processo educativo do paciente envolve medidas que o levem a conhecer todo o processo pré e póscirúrgico através das informações a ele passadas, não só pela enfermagem, mas também por toda equipe multiprofissional envolvida. Essas informações devem ser dadas ao paciente numa linguagem que ele possa entender, para facilitar sua compreensão. Embora muitos pacientes recebam essas informações relacionadas à prevenção da neoplasia de forma adequada, muitos ainda são resistentes em adotar comportamentos necessários para preveni-lo, pelos mais diversos motivos. Todos esses aspectos devem ser observados pelo enfermeiro na tentativa de favorecer a prevenção e o diagnóstico precoce da neoplasia nos pacientes transplantados renais.

Apesar de muitos aspectos do desenvolvimento das neoplasias em transplantados renais serem ainda desconhecidos, uma melhor compreensão, através de intensos estudos, do risco de neoplasia no póstransplante renal e de sua mortalidade poderão ajudar a esclarecer vários fatores no seu desenvolvimento e melhorar os resultados dos transplantes e a qualidade de vida dos pacientes. ${ }^{37}$

\section{CONCLUSÃO}

Através deste estudo puderam ser observadas questões importantes relacionadas ao desenvolvimento, nos pacientes transplantados renais, de diversos tipos de neoplasias, principalmente neoplasias incomuns na população geral, como tumores cutâneos do tipo epidermoide, Sarcoma de Kaposi, Desordens Linfoproliferativas, carcinoma cervical e colorretal, e neoplasias mais comuns, como mama e próstata.

Essas questões refletem principalmente o aumento da incidência e/ou da morbidade e mortalidade dessas neoplasias nos pacientes transplantados renais, quando comparados com a população geral. Diante disso, conclui-se que a monitorização desses pacientes quanto a manifestações precoces de acometimento de neoplasias deve fazer parte da rotina da equipe de enfermagem e multiprofissional.

Através de sua atuação como educador, o profissional enfermeiro deve priorizar ações que visem a prevenção, promoção da saúde e detecção precoce de complicações nos receptores de transplante renal, proporcionando oportunidades para o ensino e aprendizagem durante o acompanhamento pré e pós-transplante, o que pode contribuir para diminuição do risco de desenvolvimento de neoplasias nesses pacientes. Dessa forma, podese oferecer melhor qualidade de vida ao paciente, reduzindo o risco de acometimento de neoplasias e de demais complicações do pós-transplante renal.

Como limitação a esse estudo, verificou-se a indisponibilidade de publicações que tratassem das ações preventivas de enfermagem em relação a neoplasia no cuidado do paciente transplantado renal. É importante ressaltar que a maior parte dos artigos encontrados para esta pesquisa foi proveniente de estudos internacionais. Além disso, os estudos encontrados relacionados à atuação da enfermagem frente ao paciente transplantado renal, em sua maioria, abordam os cuidados de enfermagem imediatos $e$ mediatos pós-operatórios, sem levar em consideração o acompanhamento ambulatorial e necessidades de reinternação em longo prazo. Nenhum dos materiais encontrados trata das implicações para a enfermagem na prevenção e promoção da saúde relacionadas à incidência de neoplasia no seguimento do póstransplante renal, ou de órgãos em geral. Essas limitações confirmam, portanto, a necessidade de aprofundamento do tema em questão na área da enfermagem. 
Diante do exposto, pode-se apresentar como sugestão a elaboração de estudos posteriores sobre o tema em questão, para análise continuada do risco e das estratégias de prevenção da neoplasia, além da educação continuada por parte das instituições de saúde para os profissionais que cuidam de pacientes transplantados renais, visando diminuição de sua incidência e a melhora da assistência prestada a essa clientela.

\section{ABSTRACT}

Introduction: Due to the increased survival of transplant patients and the increased exposure to immunosuppressants, neoplasms become one of three leading causes of death after transplant in the last decade, and it becoming increasingly important in every organ transplant program its prevention and care. Purposes: To conduct a literature review on the development of cancer in renal transplanted patients and to identify preventive nursing interventions for the development of malignancies in renal transplanted patients. Methods: Bibliographical research with data collection of printed publications and electronic sources, on the BIREME and PubMed database. Initial search resulted in 2724 abstracts, and after verification of the proposed inclusion and exclusion criteria, 79 articles were selected for a deepen reading. The final sampling consisted of 40 articles and six printed sources, which were assessed and analyzed. Conclusion: Through this study important issues related to development in renal transplant patients, several types of cancer, especially unusual cancers in the general population, as skin tumors of squamous cell type, Kaposi's sarcoma, lymphoproliferative disorders, cervical cancer and colorectal and most common malignancies such as breast and prostate were observed. It was concluded that the monitoring of those patients searching for early signs of cancer should be part of the nursing and multidisciplinary staff routine. Nurses should prioritize actions aimed to the prevention, early health promotion and detection of complications in kidney transplanted recipients, providing teaching and learning opportunities along the pre and post-transplant, which can contribute to reducing the risk of developing neoplasia in such clientele.

Keywords: Kidney Transplantation; Neoplasia; Nursing Care.

\section{REFERÊNCIAS}

1. Associação Brasileira de Transplante de Órgãos (ABTO). Dimensionamento dos Transplantes no Brasil e em cada estado (2007-2014). Registro Brasileiro de Transplantes, 2014. Ano XX, no 4 .

2. Gallagher MP, Kelly PJ, Jardine M, Perkovic V, Cass A, Craig JC et al. Long-Term Cancer Risk of Immunosuppressive Regimens after Kidney Transplantation. J Am Soc Nephrol. 2010;21:852-8.

3. Champman JR, Webster AC, Wong G. Cancer in the Transplant Recipient. Cold Spring Harb Perspect Med. 2013;3:a015677

4. Alvares G A. Frequência de neoplasia de próstata em pacientes transplantados renais: Estudo de caso controle. (Dissertação) Faculdade de Medicina da Universidade de São Paulo. São Paulo, 2007.
5. Associação Brasileira de Transplantes de Órgãos (ABTO). Assistência de enfermagem ao paciente submetido ao transplante renal. Protocolo de cuidados de enfermagem em Transplante de Órgãos. ABTO. Brasil, 2008.

6.Carneiro, R.V. Uso de acitretina para a prevenção e tratamento de neoplasia de pele em transplantados renais: avaliação clínica histológica e imuno-histoquímica. (Dissertação) Faculdade de Medicina de São Paulo. São Paulo, 2003.

7. Hall EC, Segev DL, Engels EA. Racial/Ethnic Differences in Cancer Risk After Kidney Transplantation. Am J Transplant. 2013;13:714-20.

8. Wong G, Chapmam JR, Craig JC. Death from cancer: a sobering truth for patients with kidney transplants. Kidney Int. 2014;85:1262-4. 
9. Wu B, Wang K, Mo CB, Shen ZY. De novo malignancies in renal transplant recipients: experience at a single center in China. Int J Clin Exp Med. 2015;8:2911-16.

10. Einollahi B, Rostami Z, Nourbala MH, Lessan-Pezeshki M, Simforoosh N, Nemati E, et al. Research Paper Incidence of Malignancy after Living Kidney Transplantation: A Multicenter Study from Iran. J Cancer. 2012;3:246-56.

11. Associação Brasileira de Transplante de Órgãos (ABTO). Protocolo clínico e diretrizes terapêuticas de imunossupressão em transplante renal. ABTO. Brasil, 2010.

12. Tessari G, Naldi L, Boschiero L, Minetti E, Sandrini S, Nacchia $F$, et al. Incidence of Primary and Second Cancers in Renal Transplant Recipients: A Multicenter Cohort Study. Am J Transplant. 2013;13:214-21.

13. Engels EA, Pfeiffer RM, Fraumeni JF, Kasiske BL, Israni AK, Snyder JJ, et al. Spectrum of cancer risk among US solid organ transplant recipients. JAMA. 2011;306:1891-901.

14. Leeuwen MT, Webster AC, McCredie MRE, Stewart JH, McDonald SP, Amin J, et al. Effect of reduced immunosuppression after kidney transplant failure on risk of cancer: population based retrospective cohort study. BMJ. 2010;340:c570.

15. Instituto Nacional do Câncer (INCA). Prevenção e controle do câncer: normas e recomendações do INCA. Rev Bras Cancerol. 2002;48:317-32.

16. Ferreira FR, Ogawa MM, Nascimento LFC, Tomimori J.Epidemiological profile of nonmelanoma skin cancer in renal transplant recipients: experience of a referral center. An Bras Dermatol. 2014;89:745-50.

17. Borges-Costa J, Vasconcelos JP, Travassos AR, Guerra J, Santana A, Weigert A, et al. Cancro cutâneo em doentes com transplante renal. Acta Med Port. 2013;26:123-6.

18. Madeleine MM, Johnson LG, Daling JR, Schwartz SM, Carter JJ, Berg D, et al. Cohort Profile: The Skin Cancer After Organ Transplant Study. Int J Epidemiol. 2013;42:1669-77.

19. Keller B, Braathen LR, Marti HP, Hunger RE. Skin cancers in renal transplant recipients: a description of the renal transplant cohort in Bern. Swiss Med Wkly. 2010;140: w13036.

20. Ingvar A, Smedby HE, Lidelof B, Fernberg P, Bellocco R, Tufveson G, et al. Immunosuppressive treatment after solid organ transplantation and risk of post-transplant cutaneous squamous cell carcinoma. Nephrol Dial Transplant. 2010;25:2764-71.

21. Raeidi D, Payandeh M, Madani HS, Zare ME, Kansestani AN, Hashemian. Kaposi's Sarcoma after Kidney Transplantation: a 21-Years Experience. Int J Hematol Oncol Stem Cell Res. 2013;7:29-33.

22. Ponticelli C. Herpes viruses and tumours in kidney transplant recipients. The role of immunosuppression. Nephrol Dial Transplant. 2011;26:1769-75.

23. García PK, Buriticá JPC, Rodríguez MP, Echeverri JE, Lozano E, Benavides C. Sarcoma de Kaposi en trasplante renal, respuesta a sirolimus: reporte de caso y revisión de la literatura científica. Univ Méd Bogotá. 2011;52:209-18.

24. Morgans AK, Reshef R, Tsai DE. Posttransplant Lymphoproliferative Disorder Following Kidney Transplant. Am J Kidney Dis. 2010;55:168-80.

25. Yunus M, Aziz T, Mubarak M. Posttransplant Malignancies in Renal Transplant Recipients: 22-years Experience from a Single Center in Pakistan. Asian Pacific J Cancer Prev. 2012;13:575-8.
26.Quinlan SC, Pfeiffer RM, Morton LM, Engels EA. Risk Factors for Early-onset and Late-onset Post-transplan Lymphoproliferative Disorder in U.S. Kidney Recipients. Am J Hematol. 2011; 86(2):206-9.

27. Pietrzak B, Mazanowska N, Ekiel AM, Durlik M, Martirosian G, Wielgos $\mathrm{M}$, et al. Prevalence of high-risk human papillomavirus cervical infection in female kidney graft recipients: an observational study. Virol J. 2012;9:117.

28. Park ST, Song MJ, Park SJ, Hur SY, Lee CW. Incidence and clinicopathologic behavior of uterine cervical carcinoma in renal transplant recipients. World J Surg Oncol. 2011; 9: 72.

29. Mazanowska N, Pietrzak B, Kamiński P, Ekiel A, Martirosian G, Jabiry-Zieniewicz Z, Wielgoś M. Prevalence of cervical high-risk human papillomavirus infections in kidney graft recipients. Ann Transplant. 2013; 18:656-60.

30. Kumar D, Unger ER, Panicker G, Medvedev P, Wilson L, Humar A Immunogenicity of Quadrivalent Human Papillomavirus Vaccine in Organ Transplant Recipients. Am J Transplant. 2013;13:2411-17.

31. Instituto Nacional de Câncer (Brasil). Ações de enfermagem para o controle do câncer: uma proposta de integração ensino-serviço. Instituto Nacional de neoplasia - 3. ed. atual. amp. - Rio de Janeiro: INCA, 2008.

32. Sacoto CC, Marco SL, Mateu PB, Alba AB, Moreno JLP, Cruz JFJ. Neoplasias urológicas de novo en pacientes trasplantados renales: experiencia en 1.751 pacientes. Actas Urol Esp. 2010;34(1):88-94.

33. Hevia V, Gómez V, Nicolás VD, Álvarez S, Cañizo CG, Galeano C, et al. Development of Urologic de Novo Malignancies After Renal Transplantation. Transplant Proc. 2014; 46: 170-175.

34. Tillou X, Doerfler A, Collon S, Kleinclauss F, Patard JJ, Badet L et al. De Novo Kidney Graft Tumors: Results From a Multicentric Retrospective National Study. Am J Transplant. 2012; 12: 3308-3315.

35. Park JM, Choi MG, Kim SW, Chung IS, Yang CW, Kim YS et al. Increased Incidence of Colorectal Malignancies in Renal Transplant Recipients: A Case Control Study. Am J Transplant. 2010;10:2043-50.

36. Collins MG, Teo E, Cole RS, Chan CY, McDonald SP, RUSS $\mathrm{GR}$ et al. Screening for colorectal cancer and advanced colorectal neoplasia in kidney transplant recipients: cross sectional prevalence and diagnostic accuracy study of faecal immunochemical testing for haemoglobin and colonoscopy. BJM. 2012;345:e4657.

37. Cheung CY, Lam MF, Chu KH, Chow KM, Tsang KY, Yuen SK, et al. Malignancies After Kidney Transplantation: Hong Kong Renal Registry. Am J Transplant. 2012;12:3039-46.

38. Asch WS, Bia MJ. Oncologic Issues and Kidney Transplantation: A Review of Frequency, Mortality and Screening. Adv Chronic Kidney Dis. 2014;21(1):106-13.

39. Kato T, Kakuta Y, Yamanaka K, Okumi M, Abe T, Imamura $\mathrm{R}$, et al. Early diagnosis and treatment of breast cancer in Japanese kidney transplant recipients: a single center experience. Springerplus. 2015;4:196.

40. Kwak HY, Chae B-J, Bae J-S, Jung S-S, Song B-J. Breast cancer after kidney transplantation: a single institution review. World J Surg Oncol. 2013;11:77.

41. Mendes KDS, Roza BA, Barbosa SFF, Schirmer J, Galvão CM. Transplante de órgãos e tecidos: responsabilidades do enfermeiro. Rev Texto Contexto Enferm. 2012;21(4):945-53. 
42. Furtado AMO, Souza SROS, Oliveira BL, Garcia, CN. El enfermero asistencial y educador en una unidad de trasplante renal: un desafío. Rev Enferm Glob. 2012; 11 (27): 346-50.

43. Santos, RP, Rocha, DLB. Qualidade de vida pós-transplante renal: revisão integrativa. Enferm Nefrol. 2014;17(1):51-8.

44. Lira ALBC, Lopes MVO. Pacientes transplantados renais: análise de associação dos diagnósticos de enfermagem. Rev Gaúcha Enferm. 2010; 31(1):108-14.
45. Silva LC, Freitas TS, Maruyama SAT, Silva DRS, Silva FC. O transplante renal na perspectiva da pessoa transplantada. Ciênc Cuid Saúde. 2013; 12 (2): 356-64.

46. Association Of Women's Health, Obstetric And Neonatal Nurses. Breast Cancer Screening. Nurs Women Health. 2010; 14: 424-6. 